\title{
ROLE OF HETEROGENEOUS ASTROCYTE RECEPTOR EXPRESSION IN DETERMINING ASTROCYTIC RESPONSE TO NEURONAL DISORDERS
}

\author{
Amuti T, Ouko I, Mukonjia S, Cheruiyot I, Munguti J, Mwachaka P, Malek A
}

Correspondence to Thomas Amuti, Department of Human Anatomy, University of Nairobi, Kenya. tomamuti@gmail.com

\begin{abstract}
Following neuronal disorders, astrocytes carry out either neuroprotection or neurodegeneration. Previous authors suggest that favoring of neurodegeneration or neuroprotection by astrocytes can be due to many factors such as the influence of cytokines following their binding on their receptors on astrocytes. These receptors have however been shown to be region specific and heterogeneous. Further, research exploiting their role and influence in determining astrocytic response remains partly elucidated. A review of previous and ongoing research on these receptors would be helpful in the disclosure of astrocytic responses to neuronal disorders.
\end{abstract}

Keywords: Astrogliosis, Heterogenous astrocyte expression, Antagonistic astrocyte reaction, Nervous injury, Astrocyte mediated neurodegeneration

\section{INTRODUCTION}

Astrocytes are the most abundant macroglial cells in the central nervous system (Min et al, 2006). Their fundamental roles include nourishment of nerves (Garey, 2003), glial scar formation (Huang et al, 2014), nitric acid synthesis (Heales et al, 2004), and ion hemostasis (Ridet et al, 1997).

During neuronal disorders, astrocytes have been linked to neurodegeneration by causing leukocyte infiltration (Sonfroniew, 2000), blood brain barrier disruption (Cabezas et al, 2014) and facilitating glial scar formation (Sonfroniew, 2009), which inhibits neuronal growth. However, in other instances, they have been shown to facilitate neuroprotection by supporting axonal growth (Gage et al, 1988), aiding in the formation and release of glutathione (Belanger, 2009) and aiding in the reformation of disrupted blood brain barriers (Cabezas et al, 2014).

It has been suggested that this antagonistic astrocytic response can be due to many factors such as the influence of either neurodegenerative or neuroprotective cytokines following their binding on their receptors on the astrocytes (Yunlong et al, 2011). For example, if macroglial cells secrete a neurodegenerative cytokine such as Tumor Necrosis Factor following nervous disorders, this cytokine can influence astrocyte mediated neurodegeneration from the astrocyte upon which it binds. On the other hand, if a neuroprotective cytokine such as interleukin 6 is secreted, it can influence neuroprotective effects.

Unfortunately, it has been hinted that the receptors for the different cytokines/ligands that may influence astrocyte reaction, exhibit region specific heterogeneity (Zhang and Barres, 2010; Oberheim et al, 2012). This means that astrocytes located in one part of the brain may not necessarily share the same receptors as those located in a different part of the brain. Hence, if the receptors of a particular

Submitted $25^{\text {th }}$ October 2017. Published online $28^{\text {th }}$ February 2018. To cite: Amuti T, Ouko I, Mukonjia S et al. Role of heterogeneous astrocyte receptor expression in determining astrocytic response to neuronal disorders. Anatomy Journal of Africa. 2018. Vol 7 (1): 1169 - 1174 
cytokine/ligand are missing on the astrocytes around where it is secreted, its effects may not be observed.

Even though studies have hinted on the region specific heterogeneous nature of the receptors found on astrocytes, their influence in determining astrocyte response to neuronal disorders remains to be elucidated (Zhang and Barres, 2010).
Astrocytic reaction to nerve injury has been linked to astrocyte mediated neurodegenerative diseases like Alzheimer's Disease and Parkinson's disease whose prevalence is not only high but has been increasing (Denis et al, 1989; Lau and Breteler, 2006). Therefore, the role played by the region specific and heterogeneous nature of astrocyte receptors may be important in understanding these astrocytic mediated diseases.

\section{METHODOLOGY}

Literature was searched using Google, Hinari Scholar and PubMed for controlled and experimental studies. Search items in the databases were 'roles of astrocytes in neurodegenerative diseases', 'astrogliosis', 'glial scar formation', 'astrocyte receptors', 'cytokines', 'astrocytes' and 'reactive astrocytes'. Any additional relevant studies from the reference lists of these papers were also included. Both human studies and animal model studies were included. Out of 45 potential papers, only 28 papers were used. The 17 papers that were not used were only provided for in abstract form and this limited their use in the review.

\section{DISCUSSION}

\section{Role of the astrocyte receptors in determining astrocytic response}

\section{Heterogeneity in astrocyte receptors}

Studies on astrocytic receptors have hinted that that their expression varies from region to region (Zhang and Barres, 2010). Among the receptors that have been reported to vary are those of glutamate, erB and Il-6 (Marz et al, 1999; Zhang and Barres, 2010; Oberheim et al, 2012). For this review, these receptors were selected amongst others due to their variant expression as compared to the rest.

\section{Heterogeneity of Glutamate astrocyte receptors}

Astrocytes have been shown to express glutamate receptors that enable them to sense and respond to neuronal activity. Among the glutamate receptors that have been shown to vary depending on the brain region is the aamino-3-hydroxy-5-methyl-4-isoxazolepropionic acid (AMPA) receptors. These receptors are found mostly in astrocytes of the cerebellum, neocortex and brain stem unlike other parts of the brain (Hoft et al, 2014). AMPA receptors in cortical astrocytes are important in neuron-glia signaling as well as regulation of levels of glutamate at the synaptic cleft (Hoft et al, 2014). This regulation occurs through the absorption of excess glutamate following synaptic release of the neurotransmitter during nerve firing. This absorption prevents glutamate excito-toxicity, which is the accumulation of glutamate in the synaptic cleft (Anderson and Swanson, 2000). Excito-toxicity has been shown to occur in areas where the glutamate receptors are deficient or non-functional. Therefore, in areas such as the hippocampus, where glutamate receptors are deficient, excito-toxicity and hence neurodegeneration are prone following excess glutamate accumulation in the synaptic cleft as compared to other areas such as cerebellum, neocortex and brain stem where these glutamate receptors are abundant on the astrocytes. This is further supported by the fact 
that Alzheimer's disease (Sheldon and Robinson, 2007), which usually occurs following dysfunctional neurons in the hippocampus has been linked to glutamate toxicity. In excitotoxicity, the excess glutamate binds to N-MethylD-aspartate receptor on the post synaptic membrane of nerves following failure of its total absorption into the astrocyte (Lau and Tymianski, 2010). This has been suggested to lead to increased $\mathrm{Ca} 2+$ influx in the surrounding nerve and activation of a number of enzymes, including phospholipases, endonucleases, and proteases such as calpain. These enzymes go on to damage nerve cell structures such as components of the cytoskeleton, membrane, and DNA (Lau and Tymianski, 2010). This may explain the nervous dysfunction in Alzheimer's disease.

\section{Heterogeneity of erB astrocyte receptors}

The erB family of proteins are receptor tyrosine kinases whose role in the central nervous system is neuron to glia communication (Sharif et al, 2009). In humans, the family of erB includes erB 1 , erB 2, erB 3 and erB 4 receptors. These receptors are also identified as the HER receptors in humans (Casalini et al, 2004). They bind several ligands referred to as Epidermal Growth Factor related peptide growth factors. These stimulate the intrinsic tyrosine kinase activity of the receptors and triggers auto phosphorylation of specific tyrosine residues in the astrocyte cytoplasm (Sharif et al, 2009). These residues finally provide sites for downstream signaling molecules and eventually facilitate the effects of the growth factor. Just like the glutamate receptors, the erB receptors have been shown to vary within different brain regions with cortical astrocytes expressing erbB1, erbB2, and erbB3, whereas human hypothalamic astrocytes expressing erbB1, erbB2, and erbB4 receptors (Sharif et al, 2009). Recent data implicates insufficient ErbB signaling in neurodegenerative diseases (Gondi et al, 2009). Insufficient erB 3 and erB 4 receptors have been linked to schizophrenia whereas alterations in erB 4 has been linked to Alzheimer's disease. erB 1 and erB 2 have also been linked to pathophysiology of Parkinson's disease. Therefore, in areas expressing erB 1 and erB 2 receptors, nervous dysfunction might be common; so are the areas deficient in erB 4 receptors (Sharif et al, 2009).

\section{Heterogeneity of IL-6 astrocyte receptors}

Interleukin 6(IL-6) is a cytokine not only involved in infection and metabolic processes but also neural processes. Its role in the CNS has been reported to be antagonistic, promoting inflammation in some instances and antiinflammation in others (Scheller et al, 2011; Burton and Johnson, 2012). This antagonistic pattern has been linked to its receptors, which vary regionally. The receptors of IL 6 can be grouped into the membrane bound IL 6 receptors and the soluble IL 6 receptors. The soluble receptors are produced following cleaving of the membrane receptors immediately before the membrane-spanning region by alternative splicing or by proteolytic enzymes (Scheller et al, 2011). Research has shown that IL6 binding through its membrane receptors induces anti-inflammatory reaction. On the other hand, binding through the soluble IL 6 receptors induces pro-inflammatory reactions (Burton and Johnson, 2012). Unlike the soluble IL 6 receptors which are expressed in almost all cells, the membrane bound receptors have been shown to be highly expressed in a few cells including the astrocytes located in the cortex as compared to other areas (Marz et al, 1999). The binding of IL 6 on these areas induces the production neurotrophic factors. In the cortex, for example, IL 6 has been shown to induce production of neurotrophic factor 4 and 5 (Marz et al, 1999). These neurotrophic factors have been shown to be neuroprotective. Areas with the soluble IL 6 receptors may however be prone to astrocyte mediated neuro-degeneration. This differential receptor expression, as illustrated above, may explain the antagonistic astrocyte response to nerve injury.

These differential receptor expression in astrocytes as illustrated have been linked to the 
different embryological origins of the astrocytes (Oberheim et al, 2012).

\section{Astrocyte embryology}

Embryologically, astrocytes have been reported to differentiate from different regions (Oberheim et al, 2012). Astrocytes located in the brain have been shown to originate in the ventricular zone from radial glial intermediate cells (Voigt, 1989; Oberheim et al, 2012). These astrocytes have been reported to populate the regions of the cortex, dorsal pallium and ventral telencephalon in the developing brain (Oberheim et al, 2012). The other source of astrocytes located in the brain is the Sub ventricular zone. These astrocytes have been reported to populate most of the marginal zone during embryonic brain development, the ganglionic emminences, dorsal pallium and ventral striatum (Paterson et al, 1973; Oberheim et al, 2012). The astroblasts from the medial ganglionic emminence populate the basal ganglia and hippocampus whereas those of the lateral ganglionic emminence populate the septum and amygdala. Astrocytes from the caudal ganglionic emminence occupy the nucleus accumbens, stria terminalis and part of the hippocampus (Paterson et al, 1973; Oberheim et al, 2012). Differentiations of these astrocytes have been reported to be under regulation of many region specific molecular factors such as Gli and Nkx genes. Research has suggested that these sub types of astrocytes acquire heterogeneity in function due to this differential molecular expression during their embryology (Oberheim et al, 2012).
In the spinal cord, just like in the brain, research has also shown a difference in the origins of astrocytes. In the spinal cord, astrocytes have been reported to originate from three domains that are arranged in a dorso-ventral axis (Chaboub and Deneen, 2012). These three domains are termed p1, p2 and p3 and they have been shown to express transcription factors Pax6 and Nkx6.1, as well as Reelin and Slit (Figure 1) (Chaboub and Deneen, 2012).

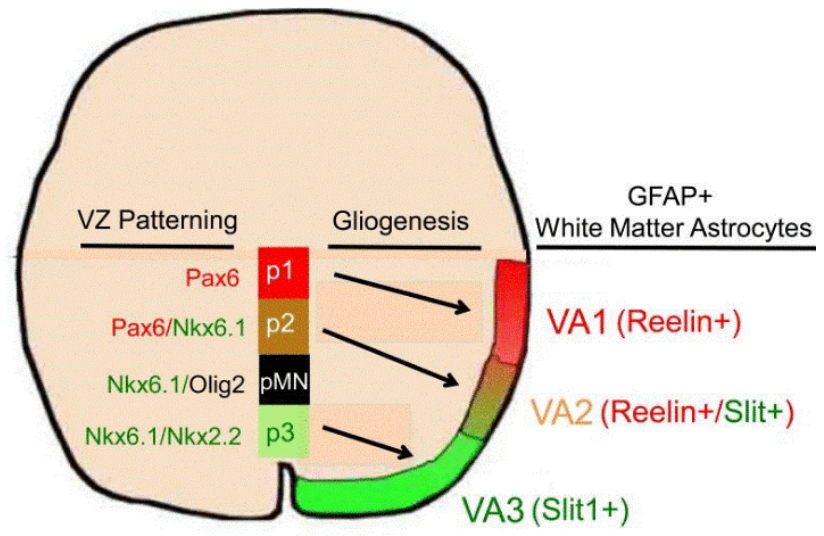

Similar to the brain, astrocytes within the spinal cord may also bear different receptor types. It is also possible that the different expression of molecular factors during their embryology in the spinal cord influences astrocyte receptor specificity, just like in the brain.

\section{Conclusion}

Astrocyte response following neuronal disorders is highly governed by distinct astrocyte receptor expression that is region specific.

\section{REFERENCES}

1. Anderson CM, Swanson RA. 2000. Astrocyte glutamate transport: Review of properties, regulation, and physiological functions. Glia; 32:1-14.

2. Burton MD, Rodney WJ. 2012. Interleukin-6 trans-signaling in the senescent mouse brain is involved in infection-related deficits in contextual fear conditioning. Brain, Behavior, and Immunity 26; 732-738.

3. Cabezas R, Ávila M, Janneth G, Ramon SB, Eliana B. 2014. Astrocytic modulation of blood brain barrier: perspectives on Parkinson's disease. Front Cell Neuroscience; 8: 211. 
4. Casalini P, Marilena VI, Enrico G. 2004. Role of HER receptors family in development and differentiation. Journal of cellular Physiology; 200: 343-350.

5. Chaboub LS, Deneen B. 2012. Developmental origins of astrocyte heterogeneity: the final frontier of CNS development. Developmental Neuroscience; 34: 379- 388.

6. Denis AE, Funkenstein H, Marilyn SA. 1989. Prevalence of Alzheimer's Disease in a community population of older persons (higher than previously reported). JAMA; 262:2551-2556.

7. Gage FH, Olejniczak P, Armstrong DM. 1988. Astrocytes are important for sprouting in the septohippocampal circuit. Exp Neurol; 102: 2-13.

8. Garey L. 2003. Role of neuroglia in central nervous system diseases. Bulletin et memoires de l'Academie royale de medicin belgium; 158:329-33.

9. Gondi CS, Dzung HD, Jeffrey DK, Meena G. 2009. MMP-2 downregulation mediates differential regulation of cell death via erbb-2 in glioma xenografts. Int Journal of Oncology; 35: 257-263.

10. Heales JR, Lam AJ, Duncan AJ, Land JM. 2004. Neurodegeneration or Neuroprotection: The pivotal role of astrocytes; Neurochemical Research; 29:513-519.

11. Höft S, Stephanie G, Gerald S, Christian S. 2014. Heterogeneity in expression of functional ionotropic glutamate and GABA receptors in astrocytes across brain regions: insights from the thalamus. Philos Trans R Soc Lond B Biology Science; 369: 20130602.

12. Huang L, Zhe BW, Qichuan Z, WeiMing Z, Bei S, Brian W, Fen S, Kunlin J. 2014. Glial scar formation occurs in the human brain after ischemic stroke. International Journal of glial scar formation occurs in the human brain after ischemic stroke medical sciences; 11- 4: 344-348.

13. Lau ML, Breteler MB. 2006. Epidemiology of Parkinson's disease. Lancet Neurology; 5: 525-35.

14. Lau ML, Tymianski M. (2010). Glutamate receptors, neurotoxicity and neurodegeneration. European Journal of Physiology; 460:525-542.

15. März P, Heese K, Dimitriades-Schmutz B, Rose-John S, Otten U. 1999. Role of interleukin-6 and soluble IL-6 receptor in region-specific induction of astrocytic differentiation and neurotrophin expression. Glia; 26:191-200.

16. Min KJ, Yang MS, Seung UK, Ilo J, Eun HJ. 2006. Astrocytes induce hemeoxygenase-1 expression in microglia: a feasible mechanism for preventing excessive brain inflammation. Journal of Neuroscience; 1-26: 1880-1887.

17. Bélanger M. 2009. The role of astroglia in neuroprotection. Dialogues Clinical Neuroscience; 11 : 281-295.

18. Oberheim NC, Steven AG, and Maiken N. 2012. Heterogeneity of astrocytic form and function. Methods Mol Biol.; 814: 23-45.

19. Paterson JA, Privat A, Ling EA, Leblond CP. 1973. Investigation of glial cells in semithin sections. III. Transformation of subependymal cells into glial cells as shown by radio autography after $\left({ }^{3} \mathrm{H}\right)$ thymidine injection into the lateral ventricle of the brain of the young rat. Journal of comparative neurology; 149: 83-102.

20. Ridet JL, Malhotra SK, Privat A, Gage FH. 1997. Reactive astrocytes: cellular and molecular cues to biological function. Trends in Neurosciences; 20:570-577.

21. Sheldon AL, Robinson MB. 2007. The Role of glutamate transporters in neurodegenerative diseases and potential opportunities for intervention. Neurochemistry Int; 51: 333-355.

22. Scheller J, Chalaris A, Schmidt-Arras D, Rose-John S. 2011. The pro- and anti-inflammatory properties of the cytokine Interleukin 6. Biochim Biophys Acta; 1813:878-88.

23. Sharif A, Duhem-Tonnelle V, Allet C. 2009, Differential erbB signaling in astrocytes from the cerebral cortex and the hypothalamus of the human brain. Glia; 57:362-79. 
24. Sofroniew VM. 2000. Astrocyte failure as a cause of CNS dysfunction. Molecular Psychiatry; 5: 230-232.

25. Sonfroniew VM. 2009. Molecular dissection of reactive astrogliosis and glial scar formation. Trends in neurosciences; 32: 638-647.

26. Voigt T. 1989. Development of glial cells in the cerebral wall of ferrets: Direct tracing of their transformation from radial glia into astrocytes. Journal of comparative neurology; 289: 74-88.

27. Yunlong MC, Tian HC, Yong Z, Jialin Z. 2011. FOXO3a inhibits TNF-a- and IL-1 $\beta$-induced astrocyte proliferation: implication for reactive astrogliosis. Glia. 59: 641-654.

28. Zhang Y and Barres BA. 2010. Astrocyte heterogeneity: an underappreciated topic in neurobiology Current Opinion in Neurobiology 20:588-594. 